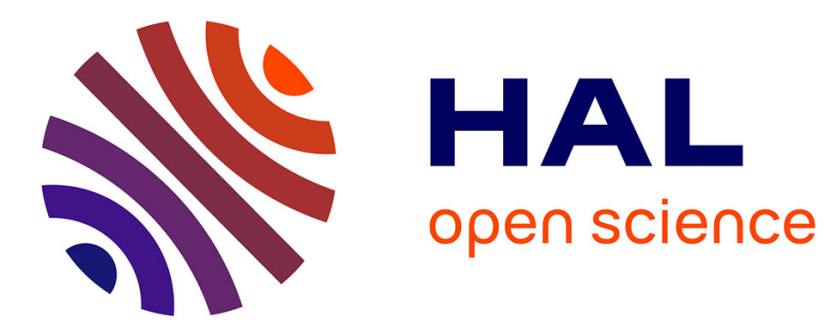

\title{
Sensitivity analysis to identify key parameters influencing infection dynamics in a pig batch
}

Amandine Lurette, Suzanne Touzeau, Matieyendou Lamboni, Hervé Monod

\section{To cite this version:}

Amandine Lurette, Suzanne Touzeau, Matieyendou Lamboni, Hervé Monod. Sensitivity analysis to identify key parameters influencing infection dynamics in a pig batch. Journal of Theoretical Biology, 2009, 258 (1), pp.43-52. 10.1016/j.jtbi.2009.01.026 . hal-00554569

\section{HAL Id: hal-00554569 https://hal.science/hal-00554569}

Submitted on 11 Jan 2011

HAL is a multi-disciplinary open access archive for the deposit and dissemination of scientific research documents, whether they are published or not. The documents may come from teaching and research institutions in France or abroad, or from public or private research centers.
L'archive ouverte pluridisciplinaire HAL, est destinée au dépôt et à la diffusion de documents scientifiques de niveau recherche, publiés ou non, émanant des établissements d'enseignement et de recherche français ou étrangers, des laboratoires publics ou privés. 


\section{Author's Accepted Manuscript}

Sensitivity analysis to identify key parameters influencing Salmonella infection dynamics in a pig batch

Amandine Lurette, Suzanne Touzeau, Matieyendou Lamboni, Hervé Monod

PII: S0022-5193(09)00030-7

DOI: doi:10.1016/j.jtbi.2009.01.026

Reference: YJTBI 5441

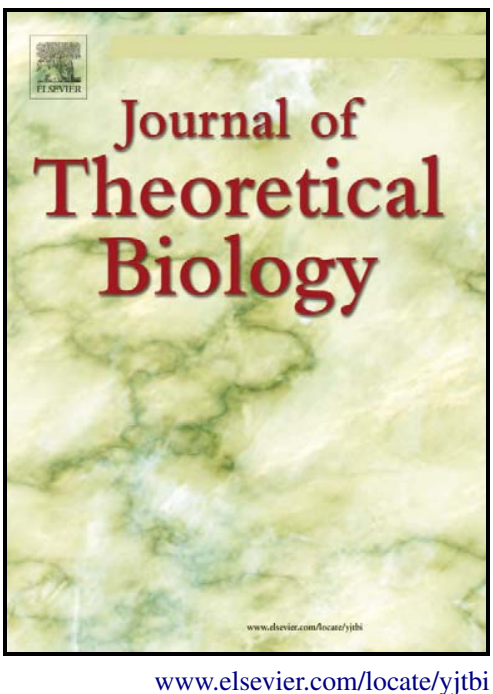

To appear in: $\quad$ Journal of Theoretical Biology

Received date: 28 May 2008

Revised date: $\quad 21$ January 2009

Accepted date: 21 January 2009

Cite this article as: Amandine Lurette, Suzanne Touzeau, Matieyendou Lamboni and Hervé Monod, Sensitivity analysis to identify key parameters influencing Salmonella infection dynamics in a pig batch, Journal of Theoretical Biology (2009), doi:10.1016/j.jtbi.2009.01.026

This is a PDF file of an unedited manuscript that has been accepted for publication. As a service to our customers we are providing this early version of the manuscript. The manuscript will undergo copyediting, typesetting, and review of the resulting galley proof before it is published in its final citable form. Please note that during the production process errors may be discovered which could affect the content, and all legal disclaimers that apply to the journal pertain. 


\title{
Sensitivity analysis to identify key parameters influencing Salmonella infection dynamics in \\ a pig batch
}

\author{
Amandine Lurette ${ }^{\mathrm{a}, \mathrm{b}, *}$ Suzanne Touzeau ${ }^{\mathrm{c}}$ \\ Matieyendou Lamboni ${ }^{\mathrm{c}}$ Hervé Monod ${ }^{\mathrm{c}}$ \\ ${ }^{\mathrm{a}}$ INRA, UMR1300 Bio-agression, Épidémiologie et Analyse de Risque, F-44300 \\ Nantes, France \\ ${ }^{\mathrm{b}}$ ENVN, UMR1300 Bio-agression, Épidémiologie et Analyse de Risque, F-44300 \\ Nantes, France \\ ' INRA, UR341 Mathématiques et Informatique Appliquées, F-78350 \\ Jouy-en-Josas, France
}

\begin{abstract}
In the context of managed herds, epidemiological models usually take into account relatively complex interactions involving a high number of parameters. Some parameters may be uncertain and/or highly variable, especially epidemiological parameters. Their impact on the model outputs then must then be assessed by a sensitivity analysis, allowing to identify key parameters. The prevalence over time is an output of particular interest in epidemiological models, so sensitivity analysis methods adapted to such dynamic output are needed.

In this paper, such a sensitivity analysis method, based on a principal component analysis and on analysis of variance, is presented. It allows to compute a
\end{abstract}


generalised sensitivity index for each parameter of a model representing Salmonella spread within a pig batch. The model is a stochastic discrete-time model describing the batch dynamics and movements between rearing rooms, from birth to slaughterhouse delivery. Four health states were introduced: Salmonella-free, seronegative shedder, seropositive shedder and seropositive carrier. The indirect transmission was modelled via an infection probability function depending on the quantity of Salmonella in the rearing room.

Simulations were run according to a fractional factorial design enabling the estimation of main effects and two-factor interactions. For each of the 18 epidemiological parameters, four values were chosen, leading to 4096 scenarios. For each scenario, 15 replications were performed, leading to 61440 simulations. The sensitivity analysis was then conducted on the seroprevalence output.

The parameters governing the infection probability function and residual room contaminations were identified as key parameters. To control the Salmonella seroprevalence, efficient measures should therefore aim at these parameters. Moreover, the shedding rate and maternal protective factor also had a major impact. Therefore, further investigation on the protective effect of maternal or post-infection antibodies would be needed.

Key words: epidemiological model, parameter uncertainty and variability, dynamic output, seroprevalence, analysis of variance

* Corresponding author. Tel.: +33(0)240687821; fax: +33(0)240687768.

Email addresses: Amandine.Lurette@vet-nantes.fr (Amandine Lurette),

Suzanne.Touzeau@jouy.inra.fr (Suzanne Touzeau), Matieyendou.Lamboni@jouy.inra.fr (Matieyendou Lamboni), Herve.Monod@jouy.inra.fr (Hervé Monod). 


\section{$1 \quad 1$ Introduction}

2 Epidemiological models are useful tools for representing infection processes.

3 They are often used to predict the spread of a pathogen in a population or to

4 assess the efficacy of control measures on that spread (Diekmann and Heester-

5 beek, 2000). In managed systems such as pig herds, they usually couple the

6 population dynamics and the pathogen transmission, to take into account the

7 relatively complex interactions between demography, management and infec-

8 tion. In general, parameters governing the herd population dynamics are well

9 known, even though they may exhibit some stochasticity due the variabil-

10 ity among individuals in the batch. Their values and range of variation can

11 be assessed from various data sources (ITP, 2000, 2006). In contrast, host-

12 pathogen interactions are not so well known. This lack of knowledge induces

13 uncertainty on the parameters involved in the herd infection processes. The

14 impact of these uncertain and variable parameters on the pathogen spread can

15 generally be best assessed by a sensitivity analysis, as analytical tractability in

16 such complex models is not ensured. A sensitivity analysis describes the effect

17 of parameter variations on model outputs. It identifies key parameters, i.e.

18 parameters that influence the outputs most, which is useful for modelling and

19 control purposes: on the one hand, improving knowledge on these parameters

20 increases the model accuracy; on the other hand, control measures acting on

${ }_{21}$ these parameters will have a greater impact on the outputs.

22 The prevalence of seropositive animals over time (seroprevalence trajectory) is

23 an output of particular interest in epidemiological models, as control measures

24 often aim at reducing the prevalence and may not be applied uniformly over

25 time. Most sensitivity analysis methods are adapted to scalar outputs (Saltelli 
et al., 2000) and cannot be applied to such dynamic outputs. However, some methods have been developed to analyse time series outputs (Campbell et al., 2006). They use classical statistical tools on variables that summarise the trajectory variability. The method developed by Lamboni et al. (2008) is based on a principal component analysis applied to the trajectories. It maximises the information that is retained from the output variability. It has already been used to analyse various agronomic models and can easily be applied to an epidemiological model.

The control of Salmonella prevalence in pig herds is a major step to reduce the pork food chain contamination. Infected pigs are asymptomatic carriers that cannot be directly detected by observation. Serological and bacteriological detection methods do exist, but they cannot be systematically applied to all pigs sent to the slaughterhouse, for practical and economical reasons. Moreover, Salmonella are ubiquitous bacteria that survive well in the environment (Murray, 2002). Hence, eliminating the bacteria in a contaminated herd is not a very realistic goal. Control measures should rather aim at reducing the prevalence level. To achieve this goal, the parameters that influence the Salmonella spread and prevalence most need to be identified. A modelling approach coupled with a sensitivity analysis can be applied to identify these key parameters.

A simulation model representing the Salmonella spread within a farrow-tofinish pig herd implementing batch-farrowing management was developed by Lurette et al. (2008a). This model details both the sow and pig dynamics (Lurette et al., 2008b) and the Salmonella infection characteristics. In such a herd, sows are distributed in groups of equal size called batches and their reproductive cycles are synchronised. At regular intervals (usually three weeks), 
52 a batch of sows is inseminated and their weaned pigs are managed together

53 as a batch until their slaughterhouse delivery. Each batch of pigs is usually

54 housed in a separate room, which is decontaminated between two batches.

55 Batches of pigs may interact, mainly through batch mixing at the end of the

56 finishing period. However, even if it occurs under normal pig growth conditions

${ }_{57}$ (i.e. adequate feed and no diseases affecting pig growth), it only concerns a

58 low number of pigs (Lurette et al., 2008b). Salmonella transmission is mostly

59 due to indirect contacts among animals via their rearing room. Infected pigs

60 shed the bacteria intermittently (Kranker et al., 2003); it is then transmitted

${ }_{61}$ to susceptible pigs by the oro-fecal route. Due to the limited contacts be-

62 tween batches, Salmonella transmission is stronger at the within-batch level.

63 So we chose to focus in this study on a single batch of pigs, from birth to

64 slaughterhouse delivery.

65 The aim of this paper is therefore to identify the parameters that contribute

${ }_{66}$ most to the variability of the Salmonella seroprevalence in a batch of pigs,

67 using a sensitivity analysis based on a principal components analysis. We will

${ }_{68}$ first describe the pig batch epidemiological model, followed by the sensitiv-

69 ity analysis. Then we will present and discuss the results obtained with this

70 method.

${ }_{71} \quad 2$ Model description

72 We developed a stochastic model to represent the Salmonella transmission 73 within a batch of pigs, from their birth to their slaughterhouse delivery. The 74 model has a discrete one-week time step, which is suited both to the repre75 sentation of the farmer task planning and to the epidemiological time scale. 
76 As the carriage of Salmonella in pigs is generally subclinic, the infection does

77 not affect the pig demography. Hence, the pig batch model and the epidemi78 ological model are first presented separately. They are then coupled by dis79 tributing the animals of the batch into the infection states. Finally, the paso rameters and initial conditions are described.

\section{2.1 Pig batch model}

82 Pigs in a batch are born simultaneously from a batch of sows at time $t=0$. ${ }_{83}$ The litter size $L$ is fixed. Pigs pass through three growing stages during their ${ }_{84}$ lifetime, denoted by $X$, corresponding to the occupancy of three rooms: (i) 85 the suckling stage, a 4 -week period from birth to weaning $\left(X=S, D_{S}=4\right)$, ${ }_{86}$ takes place with the sows in the farrowing room; (ii) the 8-week post-weaning ${ }_{87}$ period $\left(X=P W, D_{P W}=8\right)$ takes place in the post-weaning room; (iii) the 88 finishing stage takes place in the finishing room and lasts up to 14 weeks ${ }_{89}\left(X=F, D_{F}=14\right)$. The number of pigs in the batch at time $t \in\{0,1, \ldots, 25\}$ 90 is given by $P(t)$. At each time step, the number of pigs decreases because of ${ }_{91}$ mortality and, at the end of the finishing stage, because of slaughterhouse 92 delivery.

93 The mortality rate $\mu_{X}$ is assumed to be constant in each growing stage $X$ and 94 differs between stages.

95 At each delivery time (usually every two weeks), producers send groups of 96 finishers with a given slaughtering weight. As out-of-range pigs are sold at a 97 lower price, they are kept in the herd until they reach the slaughtering weight 98 or until their room needs to be emptied. Because of pig growth variability, 
99 a batch leaves the herd at different times. To represent the proportion of

All remaining pigs are delivered at the last delivery time (25 weeks of age) $t_{m}=\max _{k \in \mathbb{N}}\{k \Delta t: k \Delta t \leqslant 25\}$ because the room needs to be emptied. The proportion of pigs delivered to the slaughterhouse at time $t$, calculated from the pig population $P(t-1)$, is given by:

$$
d(t)= \begin{cases}0 & \text { if } t<22 \text { or if } t \neq k \Delta t, k \in \mathbb{N} \text { (not a delivery time), } \\ \frac{\int_{t-\Delta t}^{t} \varphi(s) d s}{1-\int_{0}^{t-\Delta t} \varphi(s) d s} & \text { if } 22 \leqslant t=k \Delta t<t_{m}, k \in \mathbb{N} \text { (delivery time) } \\ 1 & \text { if } t=t_{m} \text { (room is emptied) }\end{cases}
$$

The number of pigs at time $t$ is hence obtained from the following equations:

$$
P(t)= \begin{cases}L & \text { if } t=0 \text { (birth), } \\ \left(1-\mu_{S}\right) P(t-1) & \text { if } 0<t \leqslant 4 \text { (suckling period), } \\ \left(1-\mu_{P W}\right) P(t-1) & \text { if } 4<t \leqslant 12 \text { (post-weaning period), } \\ \left(1-\mu_{F}\right)(1-d(t)) P(t-1) & \text { if } 12<t \leqslant 25, \text { (finishing period). }\end{cases}
$$


At each time step, mortality is assumed to take place first, so slaughterhouse deliveries are given by: $D(t)=\left(1-\mu_{F}\right) d(t) P(t-1)$.

\subsection{Salmonella epidemiological model}

The epidemiological model was developed by Lurette et al. (2008a). Infected animals shed Salmonella in their feces and, hence, contaminate their local environment, i.e. their rearing room. The model represents the indirect fecal-oral transmission via encounters with the free-living Salmonella in contaminated feces on the room floor, the pen separations and the pig bodies. The free-living Salmonella in the room are modelled as a quantity of Salmonella infectious units denoted $Q(t)$ at time $t$.

Four mutually exclusive health states $(Y)$ were identified and selected from the literature and from expert opinion. A susceptible pig free of Salmonella $(Y=F)$ becomes a shedding pig $(Y=S h)$ after ingesting the bacteria. After a seroconversion delay, it is called a seropositive shedding pig $(Y=S h s)$. The shedding is intermittent. When it stops, the pig becomes a seropositive carrier pig $(Y=C s)$. The shedding can be reactivated, especially under stressful conditions. The number of pigs in each state $Y$ at time $t$ is given by $P(t, Y)$, where $P(t)=\sum_{Y} P(t, Y)$ represents the total population size. All transitions are stochastic: the number of pigs which undergo a transition between two states is drawn in a binomial law; the first parameter is the number of pigs in the initial state, the second is the individual transition probability.

Assuming homogeneous mixing, each pig is exposed to $Q(t) / P(t)$ Salmonella infectious units. Ingestion is considered to have a logarithmic effect, so the 
effective quantity of Salmonella infectious units for each susceptible pig is: $q(t)=\log (1+Q(t) / P(t))$. The probability of infection for a susceptible pig is an increasing function of $q$ with two thresholds:

$$
f(q)= \begin{cases}0 & \text { if } q=0, \\ b_{1} & \text { if } 0<q \leqslant q_{1}, \\ b_{1}+\frac{b_{2}-b_{1}}{q_{2}-q_{1}}\left(q-q_{1}\right) & \text { if } q_{1}<q \leqslant q_{2}, \\ b_{2} & \text { if } q>q_{2} .\end{cases}
$$

The probability that a shedder pig becomes seropositive derives from the seroconversion delay $\lambda_{1}$ and is equal to: $1-\exp \left(-1 / \lambda_{1}\right)$. Similarly, the probability that a seropositive shedding pig stops shedding Salmonella depends on the shedding period duration $\lambda_{2}(t)$. The shedding duration follows a lognormal distribution (with parameters $\lambda_{2_{\alpha}}$ and $\lambda_{2_{\sigma}}$, mean and standard deviation) and is recalculated at each time step $t$ to account for environmental variability. The probability that a seropositive carrier pig starts shedding again is $\beta$. As previously, the pigs which undergo a state change at each time step are drawn from binomial laws using these three individual transition probabilities.

Hence, we obtain the following system of equations, that describes the popu- 
lation evolution in the four health states:

$$
\left\{\begin{aligned}
P(t, F)= & P(t-1, F)-\operatorname{Bin}(P(t-1, F), f(q(t-1))) \\
P(t, S h)= & P(t-1, S h)+\operatorname{Bin}(P(t-1, F), f(q(t-1))) \\
& -\operatorname{Bin}\left(P(t-1, S h), 1-\exp \left(\frac{-1}{\lambda_{1}}\right)\right) \\
P(t, S h s)= & P(t-1, S h s)+\operatorname{Bin}\left(P(t-1, S h), 1-\exp \left(\frac{-1}{\lambda_{1}}\right)\right) \\
& -\operatorname{Bin}\left(P(t-1, S h s), 1-\exp \left(\frac{-1}{\lambda_{2}(t-1)}\right)\right)+ \\
& -\operatorname{Bin}(P(t-1, C s), \beta) \\
P(t, C s)= & P(t-1, C s)+\operatorname{Bin}\left(P(t-1, S h s), 1-\exp \left(\frac{-1}{\lambda_{2}(t-1)}\right)\right) \\
& -\operatorname{Bin}(P(t-1, C s), \beta) .
\end{aligned}\right.
$$

Finally, the Salmonella infectious units are subject to a degradation process at each time step with a fixed rate $\nu$. They are incremented by the infectious units shed by seropositive and seronegative shedder pigs. The resulting dynamics of the Salmonella infectious units is the following:

$$
Q(t)=(1-\nu) Q(t-1)+q_{e} P(t, S h)+q_{e} \pi_{+} P(t, S h s) .
$$

\subsection{Coupling the models}

The pig batch model and the epidemiological models are coupled as follows. At each time step, mortality and slaughterhouse delivery are first applied uniformly over the four epidemiological states in the batch. The number of 
pigs in epidemiological state $Y$ delivered to the slaughterhouse at time $t$ is therefore : $D(t, Y)=\left(1-\mu_{F}\right) d(t) P(t-1, Y) ; D(t)=\sum_{Y} D(t, Y)$ is the total number of pigs delivered. Then the epidemiological transitions are drawn and finally the number of Salmonella infectious units is updated.

Some epidemiological transitions and parameters depend on the pig growing stage. The susceptibility of piglets to Salmonella infection is considered lower during the suckling period than during the other growing stages, due to a maternal protective immunity. So, during the suckling period $S$, the probability of infection $f^{S}(q)=\varepsilon f(q)$, $\varepsilon$ being a maternal protective factor $(\varepsilon<1)$; during all other stages $X \in\{P W, F\}, f^{X}(q)=f(q)$. The shedding rate also depends on the growing stage. Let $q_{e}$ be the shedding rate of a finisher pig and $\pi_{X}$ be the shedding ratio between a pig in stage $X=S, P W$ and a finishing pig; the shedding rate in stage $X$ is $q_{e} \pi_{X}$. Finally, piglets undergo a supplementary stress at weaning, which increases the probability $\beta$ that a carrier pig starts shedding again. Denoting by $\gamma$ the value of this probability at weaning time $t=5$, at any other time, it is given by $\beta=\pi_{\beta} \gamma$, with $\pi_{\beta}<1$.

Between two batches, rooms are cleaned and disinfected, but residual Salmonella may remain in the room. When piglets are born, the room can also have been contaminated by the sows. So when the pig batch enters each rearing room, the number of Salmonella infectious units is set to a residual value denoted $Q_{X}, X$ being the corresponding stage. The number of Salmonella infectious 
units is then given by the following equations:

$Q(t)= \begin{cases}Q_{S} & \text { if } t=0 \\ (1-\nu) Q(t-1)+q_{e} \pi_{S} P(t, S h)+q_{e} \pi_{+} \pi_{S} P(t, S h s) & \text { (birth), } \\ & \text { if } 0<t \leqslant 4 \\ Q_{P W} & \text { (suckling), } \\ (1-\nu) Q(t-1)+q_{e} \pi_{P W} P(t, S h)+q_{e} \pi_{+} \pi_{P W} P(t, S h s) & \text { if } 5<t \leqslant 12 \\ & \text { if } t=5 \\ Q_{F} & \text { (post-weaning), } \\ & \text { if } t=13 \\ (1-\nu) Q(t-1)+q_{e} P(t, S h)+q_{e} \pi_{+} P(t, S h) & \text { (finishing), } \\ & \text { if } 13<t \leqslant 25 \\ & \text { (finishing). }\end{cases}$

\subsection{Model parameters and initial condition}

The demographic parameters used for the pig herd model are known from the published performance data (ITP, 2000, 2006) and are detailed in Table 1.

The epidemiological parameters, however, are not so well known. The information available is presented in Table 2 .

At the beginning of the simulation, we assume that piglets are born Salmonellafree. So $P(0, F)=L$ and $P(0, Y)=0$ for $Y \neq F$. Contamination of the batch is initiated by the residual Salmonella infectious units in the rooms $Q_{Y}$. Their 
Table 1

Demographic parameters used in the batch model.

\begin{tabular}{|c|c|c|}
\hline Name & Description & Value \\
\hline$D_{S}$ & Duration of the suckling period & 4 weeks* \\
\hline$D_{P W}$ & Duration of the post-weaning period & 8 weeks* \\
\hline$D_{F}$ & Duration of the finishing period & 14 weeks* \\
\hline$\mu_{S}$ & Mortality rate for suckling pigs & 0.01 week $^{-1 \dagger}$ \\
\hline$\mu_{P W}$ & Mortality rate for post-weaning pigs & 0.005 week $^{-1 \dagger}$ \\
\hline$\mu_{F}$ & Mortality rate for finishing pigs & 0.002 week $^{-1 \dagger}$ \\
\hline$a_{\alpha}$ & Mean age at slaughtering weight & 25.5 weeks* \\
\hline$a_{\sigma}$ & Standard deviation - & 1.5 weeks $^{*}$ \\
\hline
\end{tabular}

175

176

177

178

\section{Sensitivity analysis}

The aim of a sensitivity analysis is to define which parameters have the greatest impact on given model outputs. We chose two outputs. The first one is the Salmonella seroprevalence in the batch over time, from the piglet birth until

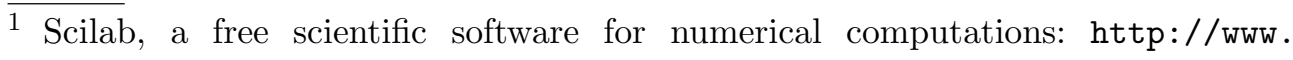
scilab.org/ 
Table 2

Epidemiological parameters used in the batch model.

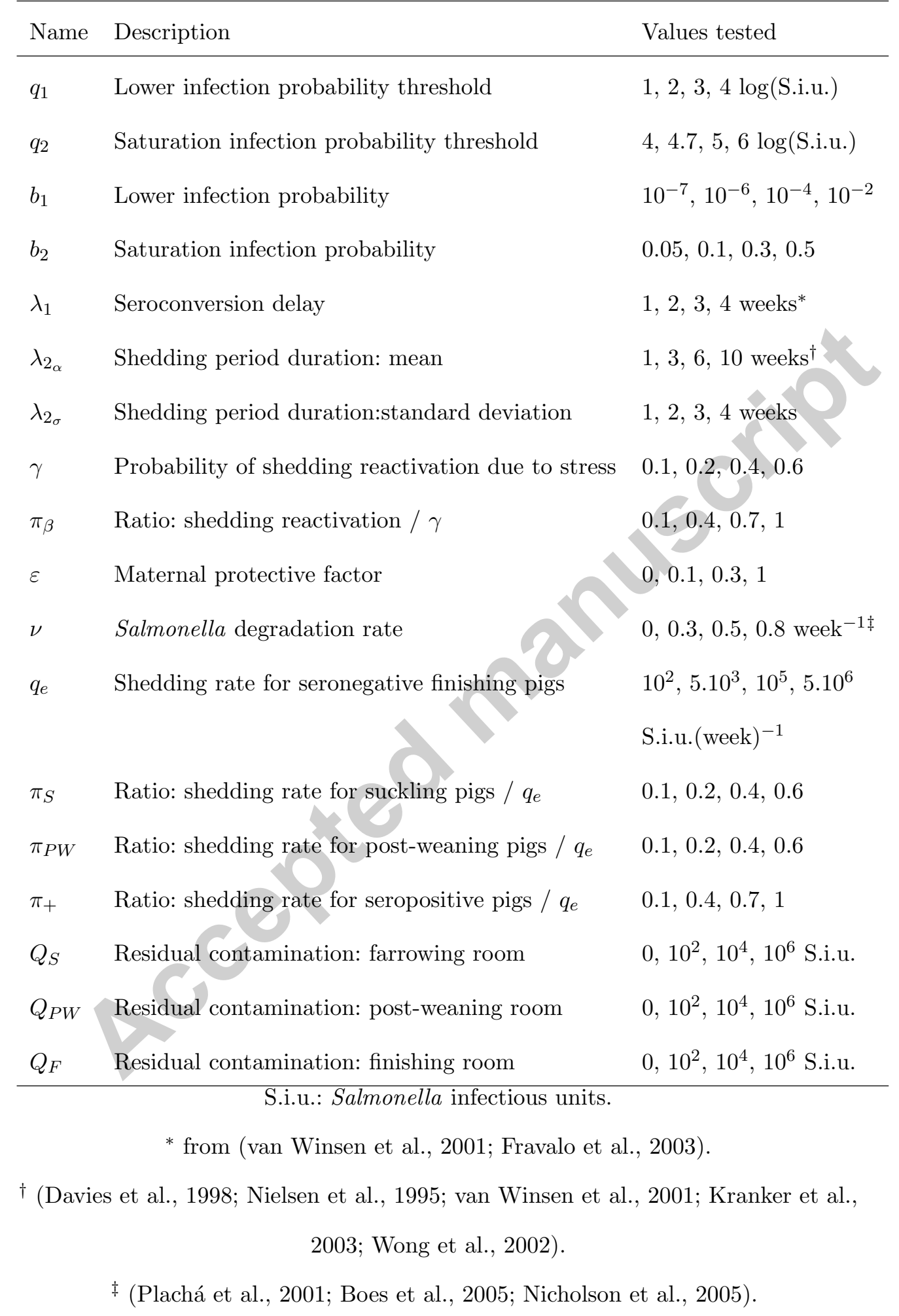


their slaughterhouse delivery. The seroprevalence corresponds to the proportion of seropositive shedding and carrying pigs in the batch. It is calculated at each time step by:

$$
\psi(t)=\frac{\sum_{Y \in S h s, C s} P(t, Y)}{P(t)} .
$$

The second output is the mean seroprevalence at slaughterhouse delivery, calculated as follows:

$$
\Omega=\frac{\sum_{t=22}^{25} \sum_{Y \in S h s, C s} D(t, Y)}{\sum_{t=22}^{25} D(t)}
$$

As the demographic parameters are well known, we chose to perform the analysis on the epidemiological parameters given in Table 2, whose values are uncertain. We used a design of experiments to define which simulations to run. Then the resulting outputs were analysed.

\subsection{Design of experiments}

The simulation model described in the previous section has 18 epidemiological parameters, also called "factors". To study the sensitivity of the outputs to these parameters, we used a factorial experimental design, with four parameter values, called "levels", per factor. As the model is stochastic, each combination of levels of factors, i.e. each parameter set or "scenario", was replicated 15 times. This number of replications was found to be largely sufficient to get robust results. It also allowed to obtain quite precise estimates of the variances between replications for each scenario.

A complete factorial design corresponds to $4^{18}$ scenarios, that is $15 \times 4^{18}$ simulations, which is not feasible. Therefore, we used a fractional factorial design (Kobilinsky, 1997; Box et al., 1978). A resolution V design is orthogonal and 
2 SAS/QC 8 User's Guide, Introduction to the FACTEX procedure: http://

it enables the estimation of main effects and two-factor interactions, assuming

that the higher order interactions are negligible. A resolution $\mathrm{V}$ design for 18 factors can be obtained with 4096 scenarios, which corresponds to 61440 simulations with 15 replications. The fractional factorial design we used was produced by the FACTEX procedure of the SAS 8.0/QC module. ${ }^{2}$ Alternative designs could have been used, in particular response surface designs (Myers and Montgomery, 2002) or space-filling designs (Fang et al., 2006). In the present study, we gave priority to orthogonal and balanced designs.

\subsection{Sensitivity analysis on the seroprevalence over time}

To analyse the seroprevalences over time $\psi(t)$ obtained with the fractional factorial design described above, we used a method developed by Lamboni et al. (2008). We only give here a short description of this method. The seroprevalence outputs can be represented in a $N \times p$ table denoted $T$, with $N=61440$ the number of simulations and $p=23$ the number of seroprevalence output times $(t=2, \ldots, 24)$. As the animals are born Salmonella-free at $t=0$ and need a week for seroconversion when they become infected, $\psi(0)=\psi(1)=0$ for each simulation; moreover, their room is emptied at $t=t_{m}=25$, so $\psi(25)=0$. Hence, these times are not included in the analysis.

A principal component analysis (PCA) with $N$ individuals and $p$ variables is performed on this table $T$. It consists in an eigenvalue decomposition of the matrix of correlations between the columns of $T, \widetilde{T}^{\prime} \widetilde{T}$, where $\widetilde{T}$ is obtained by centring and normalising each column of $T$. Let $u_{1}$ denote the first eigenvector v8doc.sas.com/sashtml/qc/chap14/index.htm 
associated with the largest eigenvalue. The first component $u_{1}$ thus obtained is the linear combination of the variables that explains the maximum of variability - or inertia - among the simulations. The second component is the second best combination to explain the variability, conditionally on being orthogonal to the first component, etc. Usually, four components are enough to explain most of the variability among the simulations. In this study, only three components were needed.

As a result of the PCA, each simulation corresponding to a line in table $T$ is given a "score" on each component. For the first component, it corresponds to the scalar product of $u_{1}$ and the corresponding line of the $T$ matrix. The scores are similarly obtained for each subsequent component. Each simulation is hence described by a scalar value called score for each component.

An ANOVA was then performed on the simulation scores for each of the first three principal components, to compare the influence of the 18 epidemiological factors on the seroprevalence. The model included all main effects and all twofactor interactions. Sensitivity indices and total sensitivities were calculated for each component $i \in\{1,2,3\}$. The sensitivity index $S I_{w}^{i}$ associated with each factorial term $w$ (main effect or interaction) is defined as the ratio between the sum of squares associated with that term and the total sum of squares. The total sensitivity $T S_{f}^{i}$ is given for each factor $f$ by the sum of the sensitivity indices corresponding to that factor; it gives the contribution of the factor and its two-factor interactions (Saltelli et al., 2000).

These indices describe the impact of the epidemiological parameters on the seroprevalence output. To assess the impact of the model stochasticity, which represents the variability among individuals in the batch, an extra sensitivity 
242 index $R S^{i}$ was calculated for each component $i$ as the ratio of the sum of

243

${ }_{244}$ This analysis was performed with the $\mathrm{R}$ software. ${ }^{3}$

245

246

247

248

249

250

251

257

258

259 ery above.

\section{Results}

256

3.3 Sensitivity analysis on the mean seroprevalence at slaughterhouse deliv-

An ANOVA was also performed to compare the influence of the 18 epidemiological parameters on the mean seroprevalence of delivered pigs $\Omega$. It was calculated for each scenario defined by the fractional factorial design described 
lences across the 4096 scenarios are represented at each time step in Fig. 1.

They show a global increase in seroprevalence over time, but they also exhibit a high variability. In $10 \%$ of the scenarios, no infection is found. In $10 \%$ of the scenarios, the batch is almost entirely infected at the slaughterhouse delivery. Box-plots at each time step and for each percentile are also drawn in Fig. 1. They show the seroprevalence variability between replicates due to model stochasticity. Each box-plot is based on the $3 \times 15$ seroprevalences associated with the 3 scenarios closest to a given percentile at a given time step. As stated previously, no infection is found for the 10th percentile. For the other percentiles, the variability due to model stochasticity appears to be quite lower than the variability due to the epidemiological factors, despite a few large outliers.

The inertia obtained for the first three principal components are $78.0 \%, 15.9 \%$ and $4.2 \%$; the next component represented less than $1 \%$ of the total variability (Fig. 2). Results are described component by component, starting with the first component.

The sensitivity indices $R S^{i}$ describing the impact of the model stochasticity for the first three components $i$ are quite low:

$$
R S^{1}=1.8 \%, \quad R S^{2}=3.6 \%, \quad R S^{3}=7.9 \% \text {. }
$$

The first principal component represents $78.0 \%$ of the total inertia. According to Fig. 3-A all time steps have comparable weights for this component, though somehow lower for the first time steps. So it corresponds more or less to an average seroprevalence effect. The most sensitive factors, which contributed to more than $20 \%$ of the variability for this component, are: $q_{1}$, the saturation 


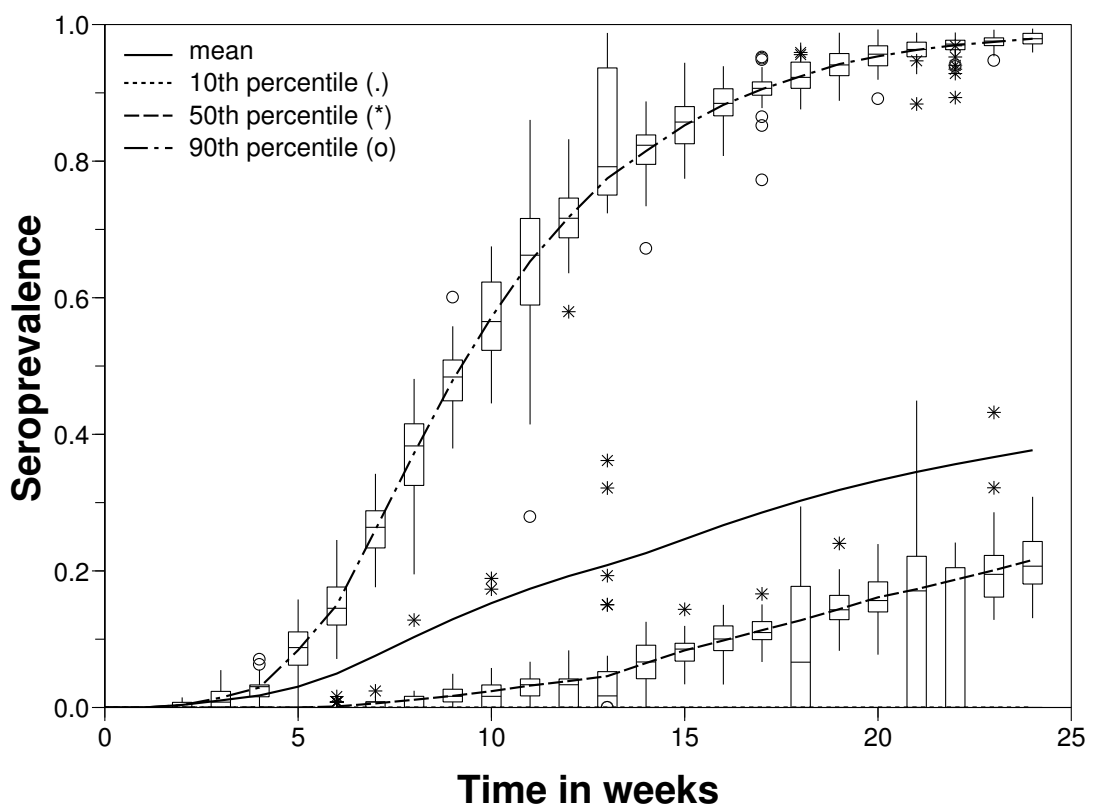

Fig. 1. Percentiles of the average seroprevalence at each time step (lines) and seroprevalence variability between replicates (boxplots). At each time step, percentiles are calculated across the 4096 seroprevalences averaged over the 15 replications per scenario: mean (plain line), $10^{\text {th }}$ percentile (dotted line, superposed with the abscissa axis), $50^{\text {th }}$ percentile (dashed line), and $90^{\text {th }}$ percentile (dash-dotted line). Each boxplot is calculated over all replications of the 3 scenarios that are most closely associated with a given percentile. In the boxplots: outliers for the $10^{\text {th }}$ percentile (.,none), $50^{\text {th }}$ percentile $(*)$, and $90^{\text {th }}$ percentile $(\circ)$. Parameters are given in Tables 1 and 2.

infection probability threshold, $b_{2}$, the saturation infection probability. The residual contamination in the farrowing room, $Q_{S}$ and in the post-weaning room $Q_{P W}$ explain more than $10 \%$ of the inertia; $q_{e}, b_{1}$ and $\varepsilon$, more than $5 \%$ each (Fig. 3-B). The cumulated sensitivity indices of the eight major main effects and two-factor interactions are higher than 70\% (Fig. 3-C). For all epidemiological parameters, the contributions of their main effect and their 


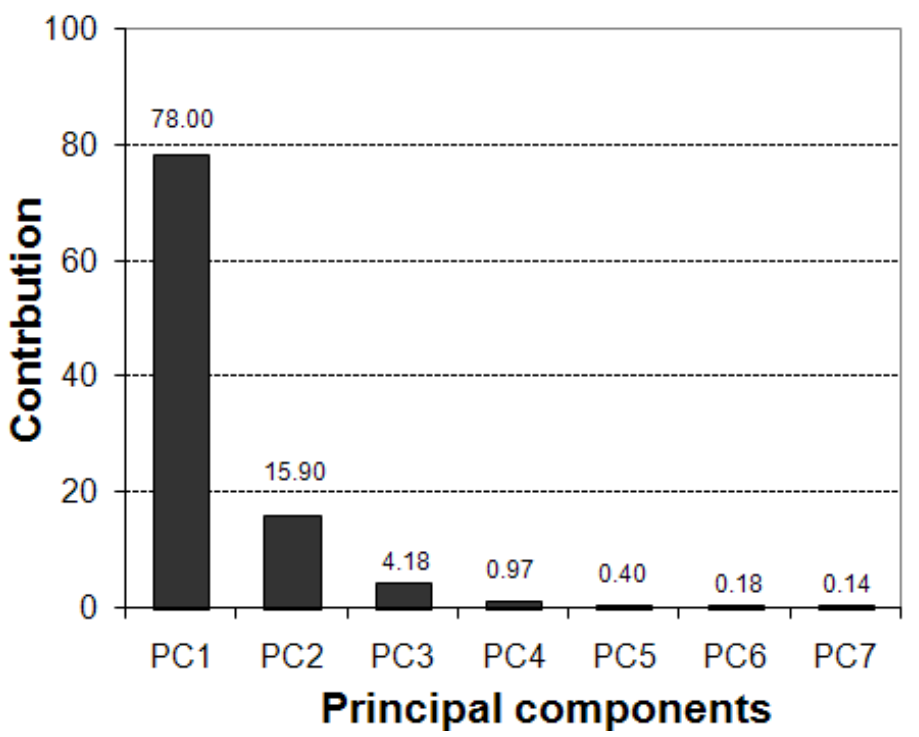

Fig. 2. Contribution to the total inertia of the seven first principal components: component contribution (black) and total inertia explained by the seven first components (grey). $P C_{X}$ is the $X^{\text {th }}$ component.

two-factor interactions are comparable. However, only one interaction appears in the eight most sensitive terms: $b_{2}: q_{1}$.

The second component only corresponds to $15.9 \%$ of the total inertia. On this component, the higher scores are attributed to simulations which exhibit higher contrasts between the beginning and the end of the simulation (Fig. 4A). The parameters explaining more variability differ from the first component (Fig. 4-B). Two parameters contribute to ca. $40 \%$ of the inertia: $\varepsilon$, the maternal protective factor, and $Q_{S}$, the residual contamination of the farrowing room. Both are involved in the early infection of piglets. $b_{2}$ and $q_{1}$, which govern the infection function, explain more than $10 \%$ of the inertia each. For all parameters, the contribution of the two-factor interactions is higher than the main effect contribution. The cumulated sensitivity indices of the eight major main factors and interactions is equal to $53 \%$; four interactions involving $Q_{S}$, 

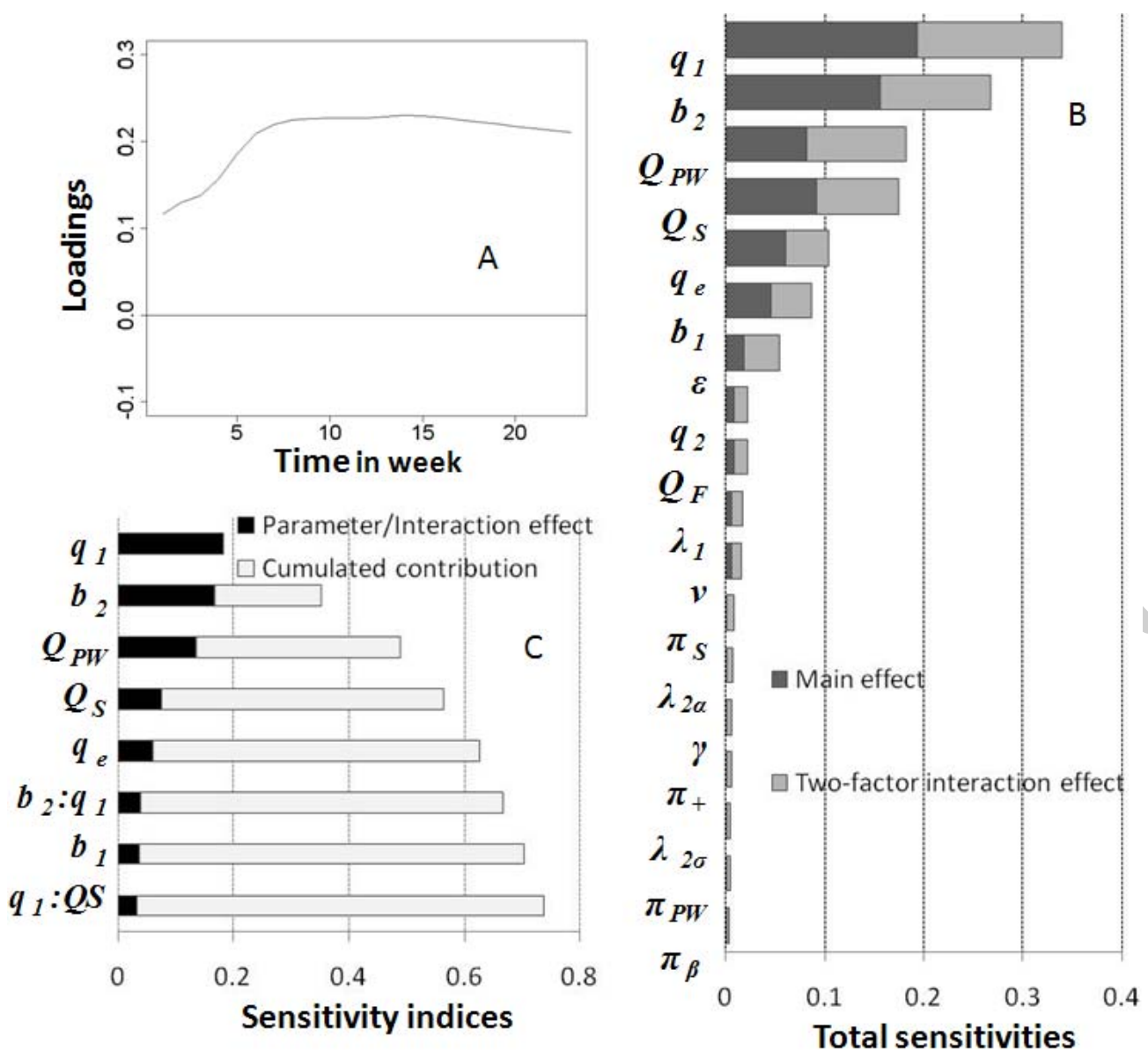

Fig. 3. Sensitivity analysis on the seroprevalence over time: results of the ANOVA performed on the simulation scores of the first component (inertia: 78.0\%). A Loadings defining the component for each time variable (in abscissa). B Total sensitivities $T S_{f}^{1}$ for the 18 epidemiological parameters $f$ ranked in descending order; sensitivities are split in main effect (black) and two-factor interactions (grey). C Sensitivity indices $S I_{w}^{1}$ of the eight main factorial terms $w$ (main effects or interactions) in descending order; individual (black) and cumulated (grey) contributions. $300 \varepsilon, q_{1}$ or $b_{2}$ are included (Fig. 4-C).

301 The third component contribution is very low: $4.2 \%$. It highlights the trajectories in which the seroprevalence during the intermediate time steps was very different from the beginning and end of the simulation (Fig. 5-A). As for 

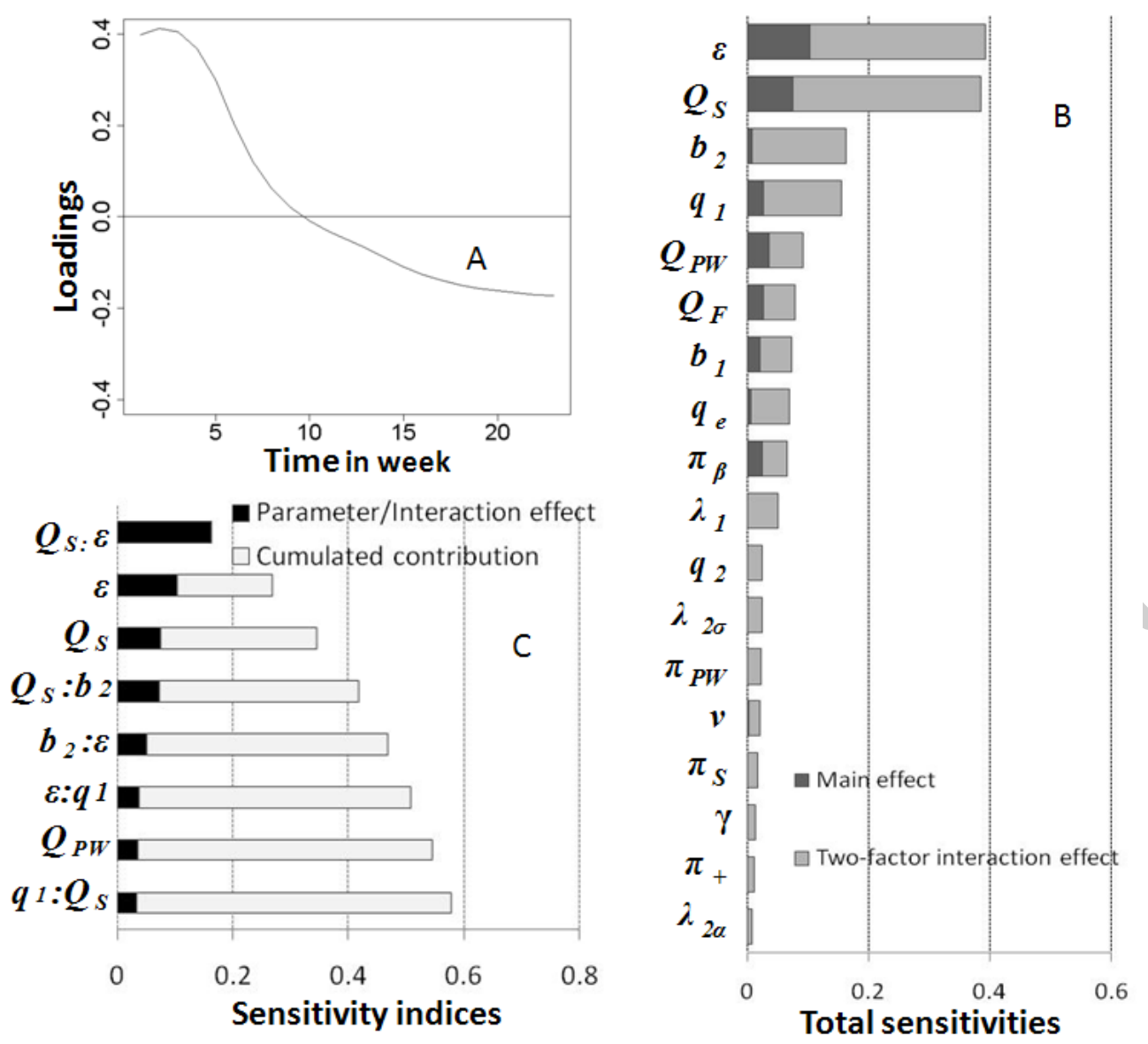

Fig. 4. Sensitivity analysis on the seroprevalence over time: results of the ANOVA performed on the simulation scores of the second component (inertia: 15.9\%).

A Loadings defining the component for each time variable (in abscissa). B Total sensitivities $T S_{f}^{2}$ for the 18 epidemiological parameters $f$ ranked in descending order; sensitivities are split in main effect (black) and two-factor interactions (grey). C Sensitivity indices $S I_{w}^{2}$ of the eight main factorial terms $w$ (main effects or interactions) in descending order; individual (black) and cumulated (grey) contributions. the second component, interactions contribute more to the inertia than major effects; they are comparable for the most influential parameter $Q_{F}$. The $Q_{F}$ total sensitivity is higher than $20 \%$ (Fig. 5-B). Parameters $Q_{F}, Q_{P W}, q_{1}$ and various two-factor interactions involving these three parameters as well as $b_{2}$, 

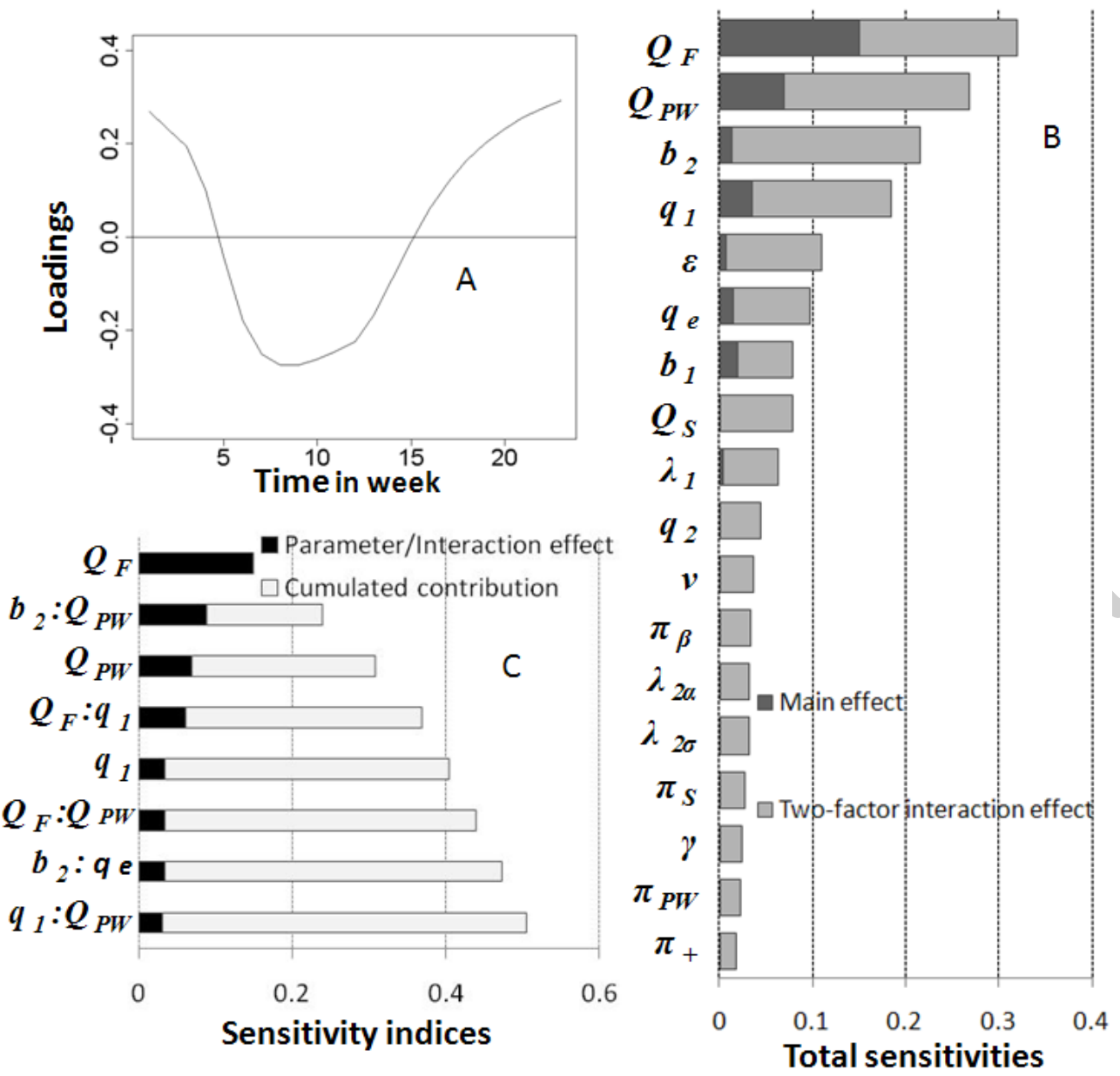

Fig. 5. Sensitivity analysis on the seroprevalence over time: results of the ANOVA performed on the simulation scores of the third component (inertia: $4.2 \%$ ). A Loadings defining the component for each time variable (in abscissa). B Total sensitivities $T S_{f}^{3}$ for the 18 epidemiological parameters $f$ ranked in descending order; sensitivities are split in main effect (black) and two-factor interactions (grey). C Sensitivity indices $S I_{w}^{3}$ of the eight main factorial terms $w$ (main effects or interactions) in descending order; individual (black) and cumulated (grey) contributions.

$q_{1}$ and $q_{e}$ explained more than $47 \%$ of the variability (Fig. 5-C). 


\subsection{Influence of factors on the seroprevalence at slaughterhouse delivery}

The terms which had a higher than $5 \%$ effect on the seroprevalence at slaughterhouse delivery are, in descending order: $q_{1}(16.1 \%), b_{2}(11.2 \%), b_{1}(5.3 \%)$, three of the four parameters governing the infection probability function, and the residual room contaminations $Q_{S}(5.3 \%)$ and $Q_{P W}(5.2 \%)$. The statistical model explains only $62 \%$ of the variability of the output.

\section{Discussion}

The model developed in this study is a simplified version of a herd model, obtained by considering an independent pig batch that only interacts with the herd through residual room contaminations. It cannot take into account batch mixing. It corresponds to an all-in/all-out management system, which is the recommended and a frequent system under normal pig growth conditions, i.e. when pig growth duration is not affected by any disease or other external factor. This batch model has allowed us to assess the Salmonella seroprevalence in a batch of pigs, from birth to slaughterhouse delivery and in the groups delivered to the slaughterhouse.

Sensitivity analyses were conducted to identify the parameters which had the greatest influence on the variability of these model outputs. The first analysis, performed on the seroprevalence over time, was more informative than the analysis performed on the seroprevalence at slaughter age. Other sensitivity analysis methods were used in previously published model describing the Salmonella spread within pig herds. Some graphically assessed the effect of infection probability variations on the seroprevalence of pigs at slaughter age 
(Hill et al., 2008). Others, in a meta-modelling approach, used a generalised linear mixed model to estimate the influence of epidemiological parameters on scalar outputs: the percentage of contaminated carcasses at the end of the slaughter process and their serological status (van der Gaag et al., 2004). In this study, we analysed the seroprevalence trajectories, estimating that the time variable would provide valuable information.

Sensitivity analyses rank sources of uncertainty and variability, according to their influence on the model outputs. These sources need not be restricted to model parameters. Here, they also included the variability among individuals in a batch, because it induces notable variations on the batch seroprevalence. Results eventually showed that this individual variability had a negligible impact on the Salmonella seroprevalence variability compared to the epidemiological parameters. The range of values tested for these parameters was chosen especially broad to account for the uncertainty on these parameters and to emphasise their impact on the output variability. However, this result was not obvious. It highlights in the present case an urgent need for more accurate estimates of the epidemiological parameter values.

Several key epidemiological parameters were identified and can be grouped in three categories. The first category consists of $q_{1}, b_{2}$ and $b_{1}$, which govern the infection probability function. These parameters are the most influential ones, as together they explained more than $40 \%$ of the first component inertia (two-factor interaction not included), the first component representing more than $78 \%$ of the total inertia. In the second category, parameters related to the room contamination, $Q_{P W}, Q_{S}$, and $Q_{F}$ are found. Their contribution to the seroprevalence variability appeared in the top eight of the first three components. The third and last category contains the Salmonella shedding 
rate for a seronegative finishing pig $q_{e}$ and the maternal protective factor $\varepsilon$. Their contribution was lower, but their impact cannot be neglected.

Infection probability function The infection probability function seems to have a major influence on the Salmonella infection dynamics. However, its four parameters do not have the same impact on the seroprevalence variability. Actually, $q_{1}$ and $b_{2}$, corresponding respectively to the lower threshold and the saturation infection probability, are the most sensitive parameters. This is consistent with a rapid growth of the quantity of Salmonella infectious units in a room. As the batch size does not vary much with time, this quantity $Q(t)$ increases with the effective quantity ingested by a pig $q(t)$. In low contaminated rooms $\left(q(t) \leqslant q_{1}\right)$, the transmission remains low. Once the room contamination exceeds a given threshold $\left(q(t)>q_{1}\right)$, the transmission increases rapidly and the infection probability reaches its saturation value $\left(f\left(q(t)=b_{2}\right)\right.$. Further investigation would be needed to define this function more accurately. The major influence of the infection probability had already been shown by van der Gaag et al. (2004): in this study, the rate of infection within a group of pigs was the most influential factor on the percentage of contaminated carcasses at the end of the slaughter process. Control measures which would reduce the probability for a susceptible pig to become infected would be very efficient. They would involve: (i) a reduction of the contact rate between susceptible pigs and all sources of infection, by implementing a strict all-in/all-out housing system for instance; and/or (ii) a reduction of the probability of infection given such a contact, by acid feed (Farzan et al., 2006) or vaccination (Roesler et al., 2006). 
Residual room contaminations The residual room contaminations were, as expected, quite important for the prevalence, especially as they were used to introduce the infection in the batch. Globally, the farrowing and post-weaning room contaminations had more impact on the seroprevalence over time. The batch was usually already infected when it entered the finishing room, so the contribution of shedding pigs to the room contamination overpowered the residual contamination. The second component brought out particularly strongly the residual farrowing room contamination. As this component emphasised trajectories exhibiting strong differences in seroprevalence between the beginning and the end of the simulation, it is quite consistent. The influence of the room contamination has been previously shown (Beloeil et al., 2003) and it highlights the need of cleaning and disinfecting the room between batches. This result is consistent with experimental results that showed that pigs removed at weaning or at 10 weeks of age from their herd and placed in Salmonella-free facilities became Salmonella-free delivered pigs (Dahl et al., 1997). Our study hence suggests that control measures should also concentrate on the residual room contamination, especially for the farrowing and the postweaning rooms. This goal could be reached by: (i) a better cleaning-disinfection process, (ii) reducing the quantity of Salmonella shed by infected pigs, and (iii) reducing the survival of these bacteria in the environment (quicker removal of contaminated feces).

Shedding Control measures reducing the shedding duration were shown to be efficient by experimentation (e.g. with vaccination (Roesler et al., 2006), or with acid feed (Farzan et al., 2006; van Winsen et al., 2001)). However, in the range of variation tested here, the shedding duration did not have a great 
impact on the seroprevalence variability. Therefore, further experiments should rather concentrate on the shedding quantity than on the shedding duration.

Maternal protection A partial maternal protection was represented by lowering the probability of infection for piglets during the first four weeks of life. The maternal protective factor had an intermediate influence in the first component and a major effect in the second component. We used a large range of variation for this factor, between 0 and 1 , because not only is its value uncertain, its existence remains to be demonstrated. The transmission from sows to piglets seems to be a critical point for the initial contamination of pig batches (Lurette et al., 2008a). However, little evidence was found in the literature to support the existence of a maternal protective factor due to passive immunity. Indeed, even if passive immunity has been found in piglets (Proux et al., 2000), no experiments have yet shown a potential protection induced by this immunity. Other authors modelling Salmonella spread within growerfinisher herds considered active immunity and represented either a complete immunity for a short period (10 days) following the infection (Hill et al., 2008), or a partial immunity for the seropositive animals (van der Gaag et al., 2004). We also attributed a partial immunity to the seropositive health states by reducing the shedding rate of seropositive animals $\left(\pi_{+}\right)$. Additional data concerning the potential protective effect of maternal antibodies or post-infection antibodies are however needed to investigate these matters further.

Hill et al. (2008) and van der Gaag et al. (2004) highlighted the importance of the infection prevalence in weaner pigs and then the importance of the transmission between sows and their piglets on the slaughterhouse delivery seroprevalence. Our study has shown that early infection, occurring between 
birth and weaning, seems to be the critical point of the Salmonella spread within a pig batch, and possibly within a herd. Literature reported that the prevalence of Salmonella at slaughter age resulted from the late infection of pigs in cases of multi-site herds (Davies et al., 1998; Nielsen et al., 1995). Actually, the serotype identified at the slaughterhouse differed from those found in the breeding and in the multiplying herds. In this study, the birth and the growth of pigs take place at the same place. Moreover, other observed data exhibit a correlation between the sow herd Salmonella status and the finishing pigs status (Nollet et al., 2005), which tends to support our findings.

\section{Conclusion}

This first work has allowed us to identify key parameters in Salmonella transmission from a model describing the Salmonella spread within a pig batch: first came parameters involved in the infection probability function, then parameters related to the room contamination, and finally the Salmonella shedding rate and maternal protective factor. We deduced from these findings that efficient control measures should focus on reducing: (i) the probability of infection, like for instance acid feed, and vaccination; and (ii) the residual room contamination, especially for the farrowing room, with a better cleaning-disinfection process, or a quicker removal of contaminated feces. This study also highlighted directions that should be further investigated to better understand the pathogen spread and control it better, such as the potential protective effect of maternal antibodies or post-infection antibodies. Given that the residual contamination of the rearing rooms and the early infection of piglets were shown o have quite an impact on the pathogen spread, it would be interesting to 
study the influence of these parameters within a farrow-to-finish herd where sows and pigs are housed together and where batch mixing can occur between batches (Hébert, 2006).

\section{References}

Beloeil, P. A., Chauvin, C., Proux, K., Rose, N., Queguiner, S., Eveno, E., Houdayer, C., Rose, V., Fravalo, P., Madec, F., 2003. Longitudinal serological responses to Salmonella enterica of growing pigs in a subclinically infected herd. Prev. Vet. Med. 60 (3), 207-226.

Boes, J., Alban, L., Bager, J., Mogelmose, V., Baggesen, D. L., Olsen, J. E., 2005. Survival of Escherichia coli and Salmonella Typhimurium in slurry applied to clay soil on a Danish swine farm. Prev. Vet. Med. 69 (3-4), 213228.

Box, G. E. P., Hunter, W. G., Hunter, J. S., 1978. Statistics for experimenters: an introduction to design, data analysis, and model building. Wiley series in probability and mathematical analysis. John Wiley \& Sons, New York.

Campbell, K., McKay, M. D., Williams, B. J., 2006. Sensitivity analysis when model output are functions. Reliab. Eng. Syst. Saf. 91 (10-11), 1468-1472.

Dahl, J., Wingstrand, A., Nielsen, B., Baggesen, D. L., 1997. Elimination of Salmonella Typhimurium infection by the strategic movement of pigs. Vet. Rec. 140 (26), 679-681.

Davies, P. R., Bovee, F. G., Funk, J. A., Morrow, W. E., Jones, F. T., Deen, J. R., 1998. Isolation of Salmonella serotypes from feces of pigs raised in a multiple-site production system. J. Am. Vet. Med. Assoc. 212 (12), 19251929.

Diekmann, O., Heesterbeek, J. A. P., 2000. Mathematical epidemiology of 
infectious diseases: model building, analysis and interpretation. Wiley Series in Mathematical and Computational Biology. Wiley.

Fang, K.-T., Li, R., Sudjianto, A., 2006. Design and modeling for computer experiments. Computer Science and Data Analysis Series. Chapman \& Hall/CRC, Boca Raton, FL.

Farzan, A., Friendship, R., Dewey, C. E., Warriner, K., Poppe, C., Klotins, K., 2006. Prevalence of Salmonella spp. on Canadian pig farms using liquid or dry-feeding. Prev. Vet. Med. 73 (4), 241-254.

Fravalo, P., Cariolet, R., Proux, K., Salvat, G., 2003. Le portage asymptomatique de Salmonella enterica par les porcs : résultats issus de la constitution d'un modèle en conditions expérimentales. In: 35èmes Journées de la Recherche Porcine. ITP, INRA, Paris, France, pp. 393-400.

Hébert, H., 27 octobre 2006. Modalités de conduite en bandes en élevage porcin: effets sur les contacts entre animaux. Thèse vétérinaire, École Nationale Vétérinaire de Nantes, France.

Hill, A. A., Snary, E. L., Arnold, M. E., Alban, L., Cook, A. J. C., 2008. Dynamics of Salmonella transmission on a British pig grower-finisher farm: a stochastic model. Epidemiol. Infect. 136 (3), 320-333.

ITP, 2000. Le mémento de l'éleveur de porcs. Institut Technique du Porc, Paris, France.

ITP, 2006. Le porc par les chiffres 2006. Institut Technique du Porc, Paris, France.

Kobilinsky, A., 1997. Les plans factoriels. In: Droesbeke, J.-J., Fine, J., Saporta, G. (Eds.), Plans d'expériences : applications à l'entreprise. Éditions Technip, Paris, Ch. 3, pp. 69-209.

Kranker, S., Alban, L., Boes, J., Dahl, J., 2003. Longitudinal study of Salmonella enterica serotype typhimurium infection in three danish farrow- 
to-finish swineherds. J. Clin. Microbiol. 41 (6), 2282-2288.

Lamboni, M., Makowski, D., Monod, H., Apr. 2008. Multivariate global sensitivity analysis for discrete-time models, technical report available: http://www.inra.fr/miaj/public/nosdoc/rap2008-3.pdf.

Lurette, A., Belloc, C., Touzeau, S., Hoch, T., Ezanno, P., Seegers, H., Fourichon, C., Apr. 2008a. Modelling salmonella spread within a farrow-to-finish pig herd, veterinary Research.

Lurette, A., Belloc, C., Touzeau, S., Hoch, T., Seegers, H., Fourichon, C., 2008b. Modelling batch farrowing management within a farrow-to-finish pig herd: influence of management on contact structure and pig delivery to the slaughterhouse. Animal 2 (1), 105-116.

Murray, C., 2002. Mathematical epidemiology of infectious diseases: model building, analysis and interpretation. Wiley Series in Mathematical and Computational Biology. Wiley.

Myers, R. H., Montgomery, D. C., 2002. Response surface metholology : process and product optimization using designed experiments, 2nd Edition. Wiley Series in Probability and Statistics. Wiley-Interscience, New York.

Nicholson, F., Groves, S. J., Chambers, B. J., 2005. Pathogen survival during livestock manure storage and following land application. Bioresour. Technol. $96(2), 135-143$.

Nielsen, B., Baggesen, D., Bager, F., Haugegaard, J., Lind, P., 1995. The serological response to Salmonella serovars typhimurium and infantis in experimentally infected pigs. The time course followed with an indirect antiLPS ELISA and bacteriological examinations. Vet. Microbiol. 47 (3-4), 205218.

Nollet, N., Houf, K., DeWulf, J., Duchateau, L., Zutter, L. D., Kruif, A. D., Maes, D., 2005. Distribution of Salmonella strains in farrow-to-finish pig 
herds: A longitudinal study. J. Food Prot. 68 (10), 2012-2021.

Plachá, I., Venglovský, J., Sasáková, N., Svoboda, I. F., 2001. The effect of summer and winter seasons on the survival of Salmonella typhimurium and indicator micro-organisms during the storage of solid fraction of pig slurry. J. Appl. Microbiol. 91 (6), 1036-1043.

Proux, K., Houdayer, C., Humbert, F., Cariolet, R., Rose, V., Eveno, E., Madec, F., 2000. Development of a complete ELISA using Salmonella lipopolysaccharides of various serogronps allowing to detect all infected pigs. Vet. Res. 31 (5), 481-490.

Roesler, U., Heller, P., Waldmann, K., Truyen, U., Hensel, A., 2006. Immunization of sows in an integrated pig-breeding herd using homologous inactivated Salmonella vaccine decreases the prevalence of Salmonella typhimurium infection in the offspring. J. Vet. Med. B. 53 (5), 224-228.

Saltelli, A., Chan, K., Scott, E. M. (Eds.), 2000. Sensitivity Analysis. Wiley series in Probability and Statistics. John Wiley \& Sons.

van der Gaag, M. A., Vos, F., Saatkamp, H. W., van Boven, M., van Beek, P., Huirne, R. B. M., 2004. A state-transition simulation model for the spread of Salmonella in the pork supply chain. Eur. J. Oper. Res. 156 (3), 782-798. van Winsen, R. L., van Nes, A., Keuzenkamp, D., Urlings, H. A. P., Lipman, L. J. A., Biesterveld, S., Snijders, J. M. A., Verheijden, J. H. M., van Knapen, F., 2001. Monitoring of transmission of Salmonella enterica serovars in pigs using bacteriological and serological detection methods. Vet. Microbiol. 80 (3), 267-274.

Wong, D. M. A. L. F., Hald, T., van der Wolf, P., Swanenburg, M., 2002. Epidemiology and control measures for Salmonella in pigs and pork. Liv. Prod. Sci. 76 (3), 215-222. 